\title{
Typical electronegative electroretinography and nyctalopia as a presenting feature of systemic malignant melanoma
}

This article was published in the following Dove Press journal: International Medical Case Reports Journal

\author{
Abby Vercio \\ KV Chalam \\ Timothy Winter \\ Department of Ophthalmology, Loma \\ Linda University School of Medicine, \\ Loma Linda, CA, USA
}

\begin{abstract}
We report a unique case of a female who presented with unilateral disk edema, melanoma-associated retinopathy symptoms, and suggestive electroretinography findings preceding a diagnosis of metastatic melanoma of the pelvis. A 63-year-old female presented with complaints of seeing shimmering lights and nyctalopia, and underwent an extensive ophthalmological and electrophysiological examination. Bestcorrected visual acuity was $20 / 20$ in both eyes. Visual fields showed relative central scotomata and concentric narrowing. Slit-lamp and fundus examinations were normal. Rod-specific electroretinography responses were severely reduced, with electronegative maximal combined rod-cone responses and delayed cone responses with normal amplitude. Melanoma-associated retinopathy was suspected. Extensive systemic and internal evaluation revealed occult metastatic melanoma of the pelvis of unknown primary site Keywords: nyctalopia, ERG, S cone syndrome, melanoma
\end{abstract}

\section{Introduction}

Melanoma-associated retinopathy (MAR), a rare paraneoplastic disorder, often targets the rod-response system of the eye. ${ }^{1,2}$ Consequentially, abnormal night vision and photopsia are the most common presenting symptoms. ${ }^{1-5}$ Patients usually have an established diagnosis of cutaneous melanoma (with non ocular metastasis) years prior to the onset of ocular symptoms. ${ }^{3}$ Electroretinography (ERG) testing with characteristic "electronegative" appearance, preservation of the dark-adapted a-wave, and reduction of the b-wave confirms the diagnosis. Though an established entity, MAR remains an infrequent phenomenon and reported rarely. ${ }^{3}$ We report a case of MAR with typical ERG findings in a female without a history of melanoma. Further investigations (under the direction of a neuro-oncology service) detected primary melanoma in the pelvis with metastasis (responsible for systemic and ocular findings).

\section{Case report}

A 63-year-old female with a history of hyperlipidemia and tobacco use presented for 4 days of sudden, painless "shimmering lights" in her left eye associated with nyctalopia. Best-corrected visual acuity was 20/20-I in the right eye and 20/20 in the left. Pupils, color vision, extraocular movement, confrontational fields, and anterior-segment examination were normal.
Correspondence: KV Chalam Department of Ophthalmology, Loma Linda University School of Medicine, I 1370 Anderson Street - suite 2025, Loma Linda, CA II370, USA

Email kchalam@llu.edu 

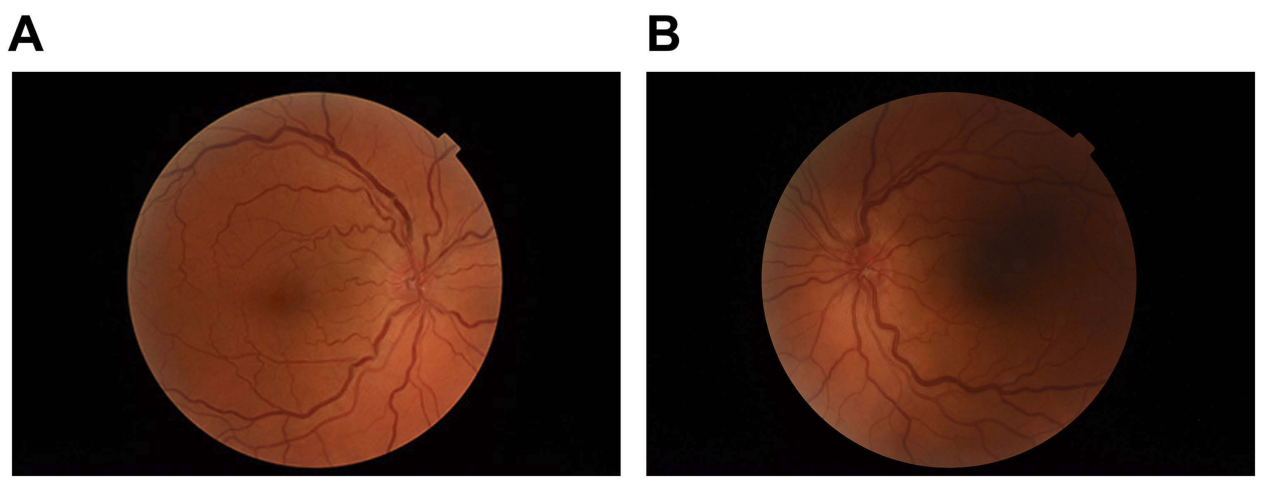

Figure I Fundus photographs demonstrating trace inferior disk edema on OD (A) and bilateral vessel tortuosity (B).

Fundoscopy revealed a cup:disk ratio of 0.15 in both eyes, 1+ inferior disk edema in the left eye, and vascular tortuosity in both eyes (Figure 1). A B-scan was negative for drusen of optic nerves. Optical coherence tomography of the nerve-fiber layer showed thickening in the right eye (121 right, 111 left, Figure 2). Humphrey visual field 24-2 showed an enlarged blind spot with superior altitudinal defect in the right eye and a superior arcuate defect in the left (Figure 3). Complete blood count, erythrocyte-sedimentationrate, and CRP were normal. She was initially diagnosed with non arteritic ischemic optic neuropathy and was started on oral prednisone $0.5 \mathrm{mg} / \mathrm{kg}$.

Two months after initiation of treatment, her night vision continued to deteriorate. Repeat Humphrey visual field 24-2 showed worsening peripheral suppression in both eyes (Figure 4). Multifocal and full-field ERG revealed intact macular cone function and abnormal rod-system response to scotopic flash with an electronegative response (Figure 5) suggestive of MAR. She was immediately referred to the neuro-oncology service for evaluation. Head computed tomography and brain magnetic resonance imaging were normal. Whole-body positron-emission tomography showed a $6.4 \times 4.4 \mathrm{~cm}$ hypermetabolic focus at the left pelvic wall with additional lesions (asymptomatic) of the left distal femur, left gastrocnemius, and left iliacus (Figure 6). Magnetic resonance imaging of the abdomen/pelvis confirmed a left pelvic wall mass (Figure 7). The gynecology-oncology department performed an excisional biopsy of the mass. Histo pathological examination revealed a poorly differentiated malignancy with melanocytic differentiation (Figure 8), consistent with metastatic melanoma.
Further imaging revealed spread to pulmonary nodules, right intra parotid nodules, a mass on the left dorsal foot, and right carotid-sheath nodules. The patient was commenced on pembrolizumab for treatment of her metastatic melanoma. repeated four times over 6 months. The patient was followed for 14 months. Repeat positron-emission tomography-computed tomography 7 months after initial diagnosis revealed marked decreases in the size and activity of the left pelvic wall mass. Activity in pulmonary nodules, right intra parotid nodules, left dorsal foot thickening, and right carotid-sheath nodules remained stable. She remained asymptomatic, except for intermittent headaches and fluctuating visual fields.

\section{Discussion}

MAR is a paraneoplastic process involving autoantibodies against "on" bipolar cells of the rod system. ${ }^{1,2}$ Though the target antigen has historically been elusive, TRMP1, present on both melanocytes and bipolar cells, has been implied in many cases. Disruption of bipolar cell function prevents appropriate neuroretinal transmission from photoreceptors to the inner retina, leading to an effective decrease in vision. Unlike other paraneoplastic processes, MAR is usually diagnosed as a late feature of diagnosed melanoma., ${ }^{1,3}$ With a latency period averaging 3.6 years from diagnosis of the primary neoplasm, the onset of MAR indicates the presence of metastasis. ${ }^{3}$ Age of onset is commonly during the sixth decade of life, with a male:female ratio of $4.7: 1^{3}$

Typical patient presentation includes acute onset of night blindness, shimmering, flickering, or pulsating 
RNFL Single Exam Report OU

SPECTRALIS $®$ Tracking Laser Tomography

THEIDELEETட

\begin{tabular}{ll} 
Sex: & F \\
Exam.: & Apr/27/2017 \\
\hline
\end{tabular}

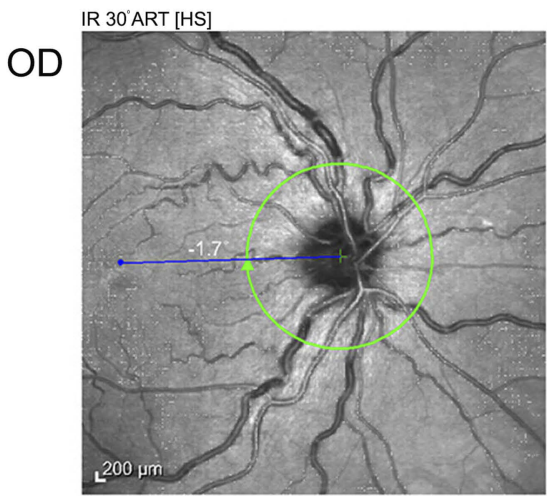

OCT ART (100) Q: 22[HS]
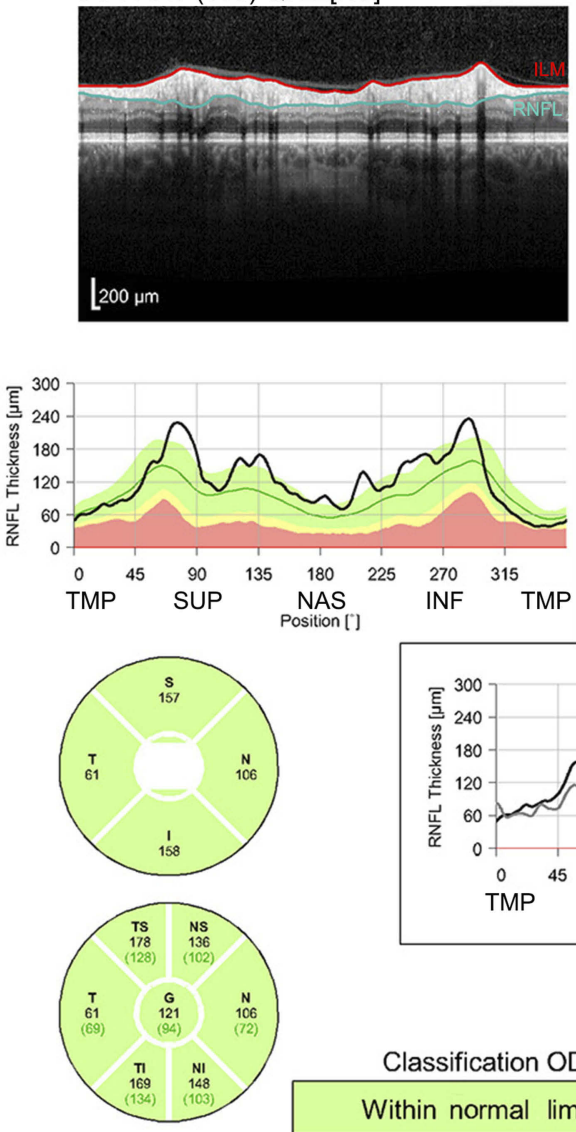

Classification OD

Within normal limits

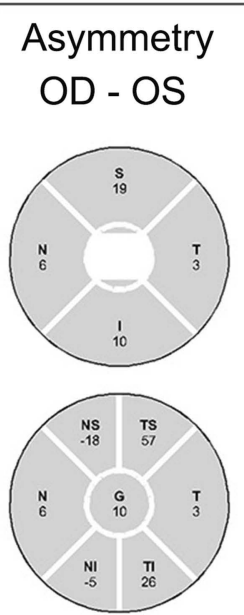

OCT ART (100) Q: 23[HS]

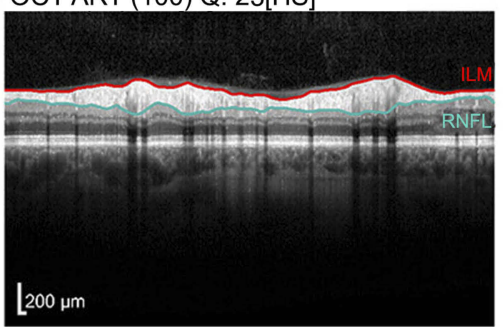

Within normal limits

Borderline $(P<0.05)$

Outside normal limits

Outside ,
$(P<0.01)$

OD

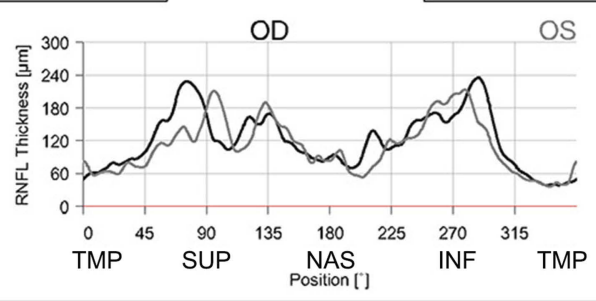

Classification OS

Within normal limits

Position []

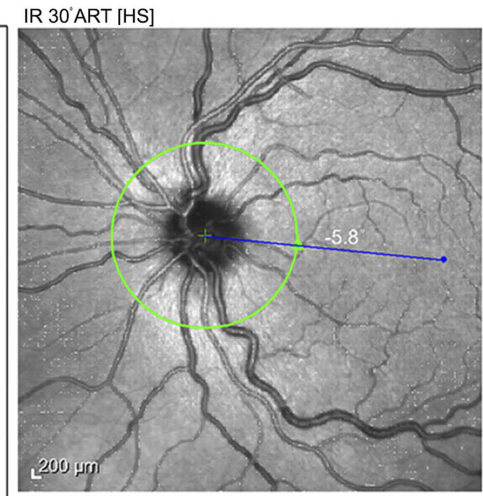

OS

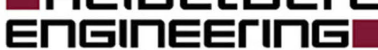
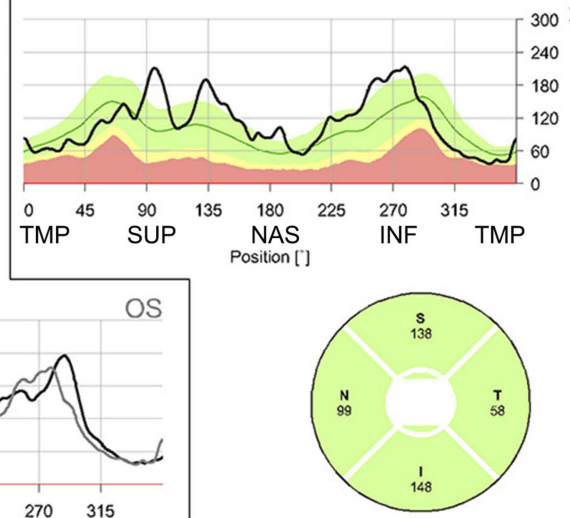

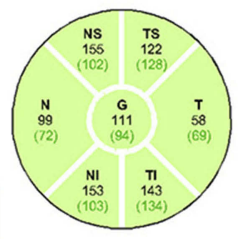

Warning: Classification results valid for Caucasian eyes only.

Notes:

Date: $4 / 27 / 2017 \quad$ Signature:

Software Version: 6.3.4 www.HeidelbergEngineering.com

RNFL Single Exam Report OU

Figure 2 Initial optical coherence tomography of nerve-fiber layer showing thickening OD (I2I) corresponding to inferior edema. 
A

Single field analysis

Central 24-2 threshold test

Fixation monitor: blind spot

Fixation target: central

Fixation losses: $3 / 13 x x$

False POS errors: $18 \% x x$

False NEG errors: $16 \%$

Test duration: 06:07

Fovea: $20 \mathrm{~dB}$

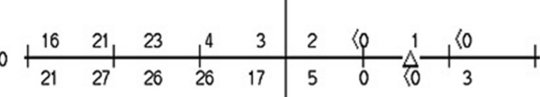

22

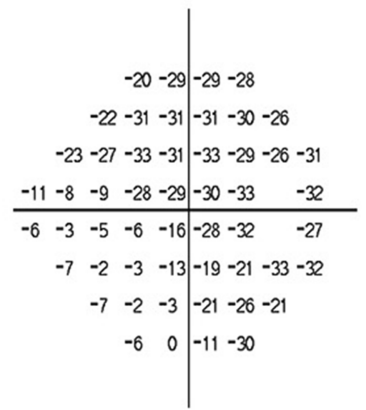

Total deviation

प1

in...…

ㅁㅁㅁㅁ

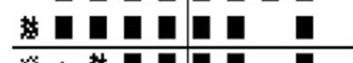

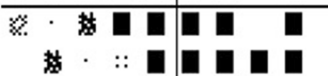

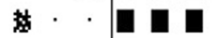

样

口
Stimulus: III, white

Strategy: SITA-fast
Background: 31.5 ASB
Eye: Right

$\begin{array}{ll}\text { Pupil diameter: } & \text { Date: 04-27-2017 } \\ \text { Visual acuity: } & \text { Time: 4:34 PM } \\ \text { RX: +6.25 DS -1.50 DC X 88 } & \text { Age: } 63\end{array}$
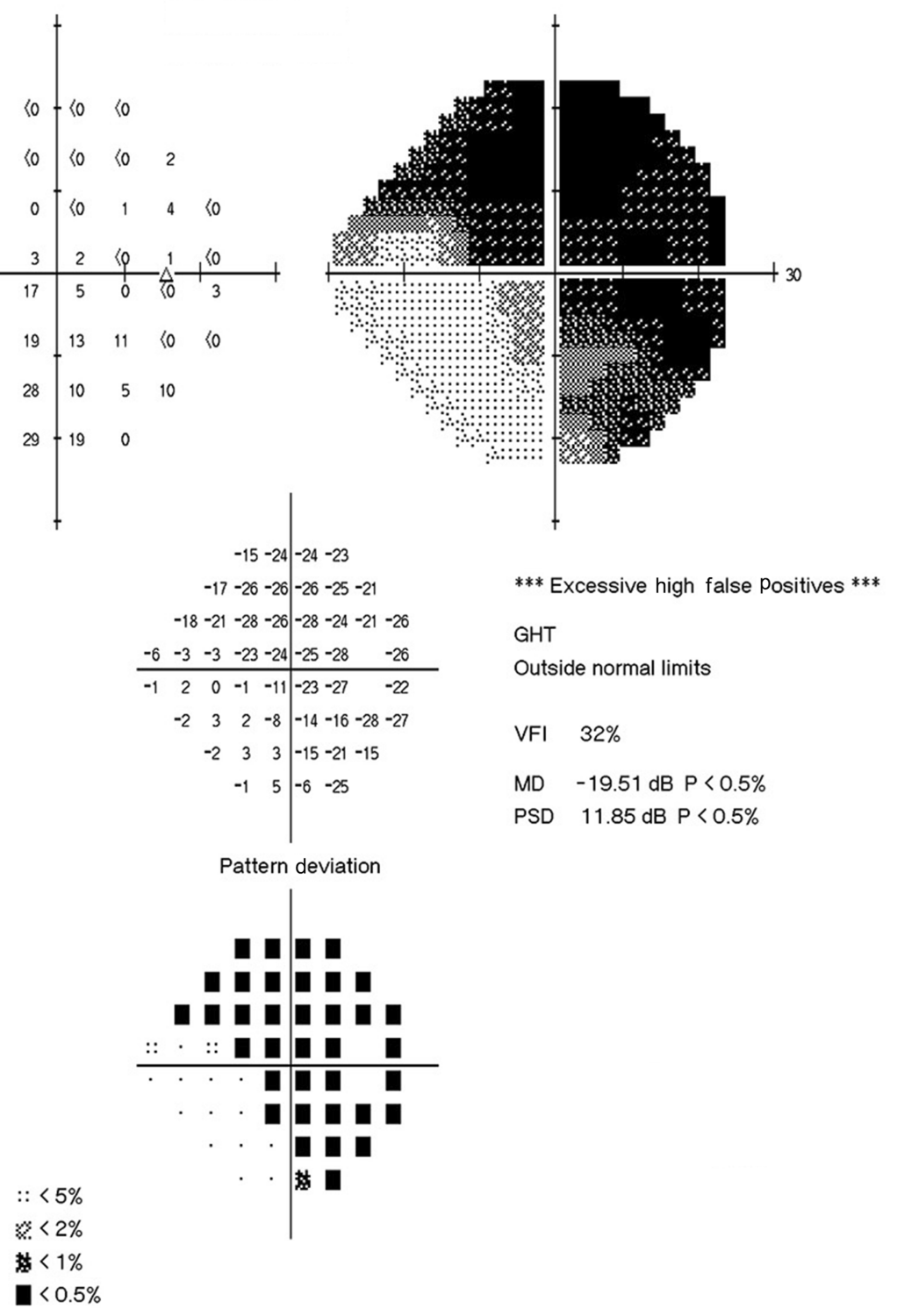

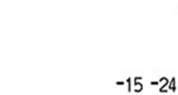

$-17-26-26-26-25-21$

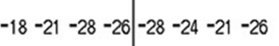

\begin{tabular}{lllll|lll}
-6 & -3 & -3 & -23 & -24 & -25 & -28 & -26 \\
\hline-1 & 2 & 0 & -1 & -11 & -23 & -27 & -22
\end{tabular}

\begin{tabular}{llll|llll}
-2 & 3 & 2 & -8 & -14 & -16 & -28 & -27
\end{tabular}

$\begin{array}{llllll}-2 & 3 & 3 & -15 & -21 & -15\end{array}$

\begin{tabular}{ll|ll}
-1 & 5 & -6 & -25
\end{tabular}

Pattern deviation
*** Excessive high false Positives ***

GHT

Outside normal limits

VFI $32 \%$

MD $\quad-19.51 \mathrm{~dB} P<0.5 \%$

PSD $\quad 11.85 \mathrm{~dB} P<0.5 \%$

() 2007 Carl zeiss meditec

HFA II 750-10734-4.2.2/4.2.2

Figure 3 Continued. 
B

Single field analysis

Central 24-2 threshold test

Fixation monitor: blind spot

Fixation target: central

Fixation losses: $12 / 12 x x$

False POS errors: $4 \%$

False NEG errors: $8 \%$

Test duration: 04:10

Fovea: $27 \mathrm{~dB}$
Eye: left

Stimulus: III, white Background: 31.5 ASB

Strategy: SITA- fast
Pupil diameter:
Visual acuity:
Date: 04-27-2017
RX: +6.50 DS -1.25 DC X 102
Time: 4:42 PM

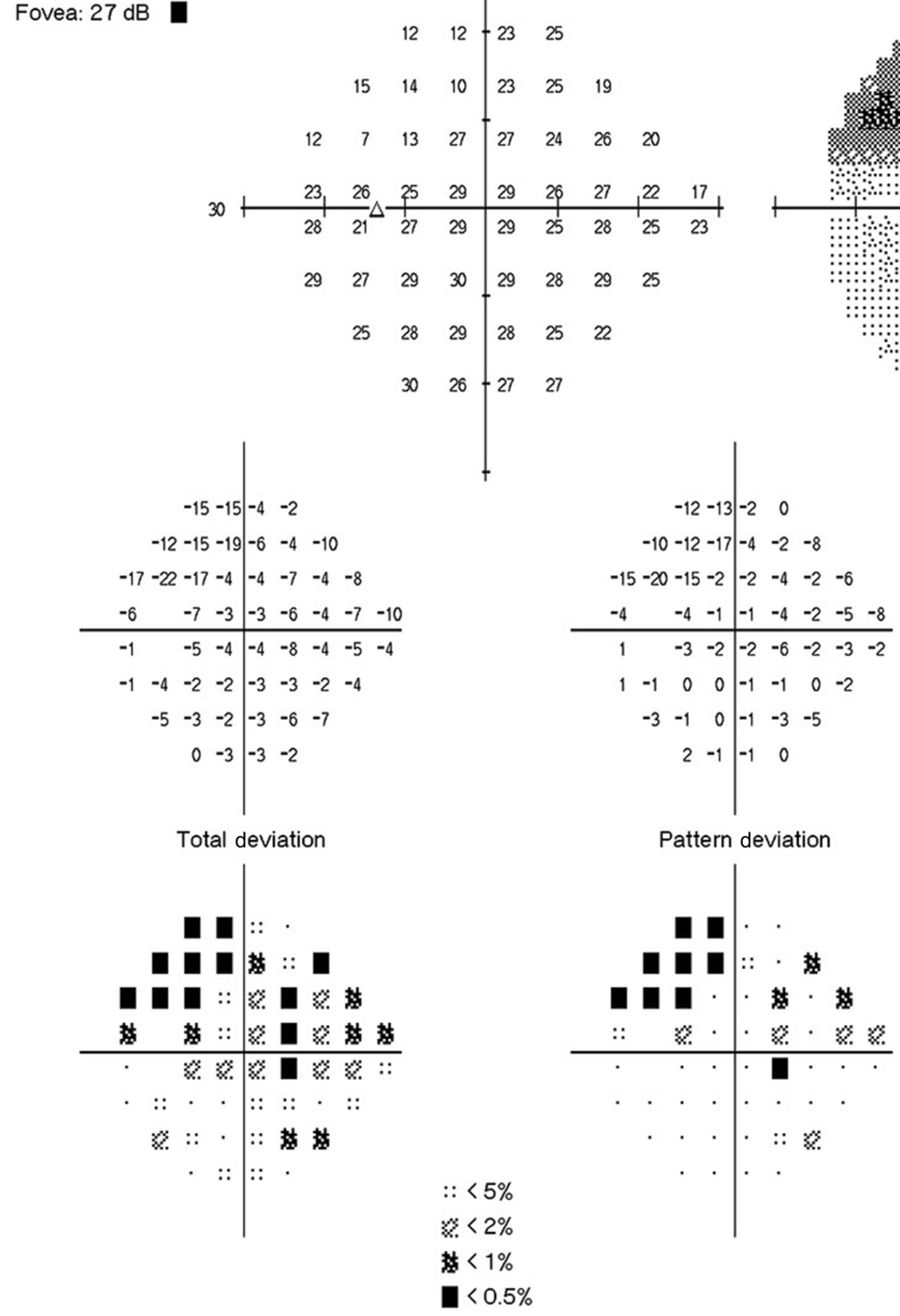

*** Low test reliability $* * *$

GHT

Outside no mal limits

VFI $89 \%$

MD $\quad-5.80 \mathrm{~dB} P<1 \%$

PSD $\quad 4.84 \mathrm{~dB} P<0.5 \%$

Pattern deviation

(1) 2007 Carl zeiss meditec

HFA || 750-10734-4.2.2/4.2.2

Figure 3 Humphrey visual fields, 24-2.

Notes: Initial tests (A). Right eye (right) with enlarged blind spot and superior altitudinal defect; left eye (left) with superior arcuate defect. Two months later (B) after treatment with prednisone. Right eye (right) with improvement centrally and dense superior and inferior arcuate defects; left eye (left) with worsening of superior and new inferior arcuate defects. 
A

Single field analysis

Eye: left

Central 24-2 threshold test

Fixation monitor: blind spot

Fixation target: central

Fixation losses: 0/16

False POS errors: $2 \%$

False NEG errors: $14 \%$

Test duration: 05:53

Fovea: $28 \mathrm{~dB}$

Stimulus: III, white Background: 31.5 ASB

Strategy: SITA- fast

Fovea: 28 dB
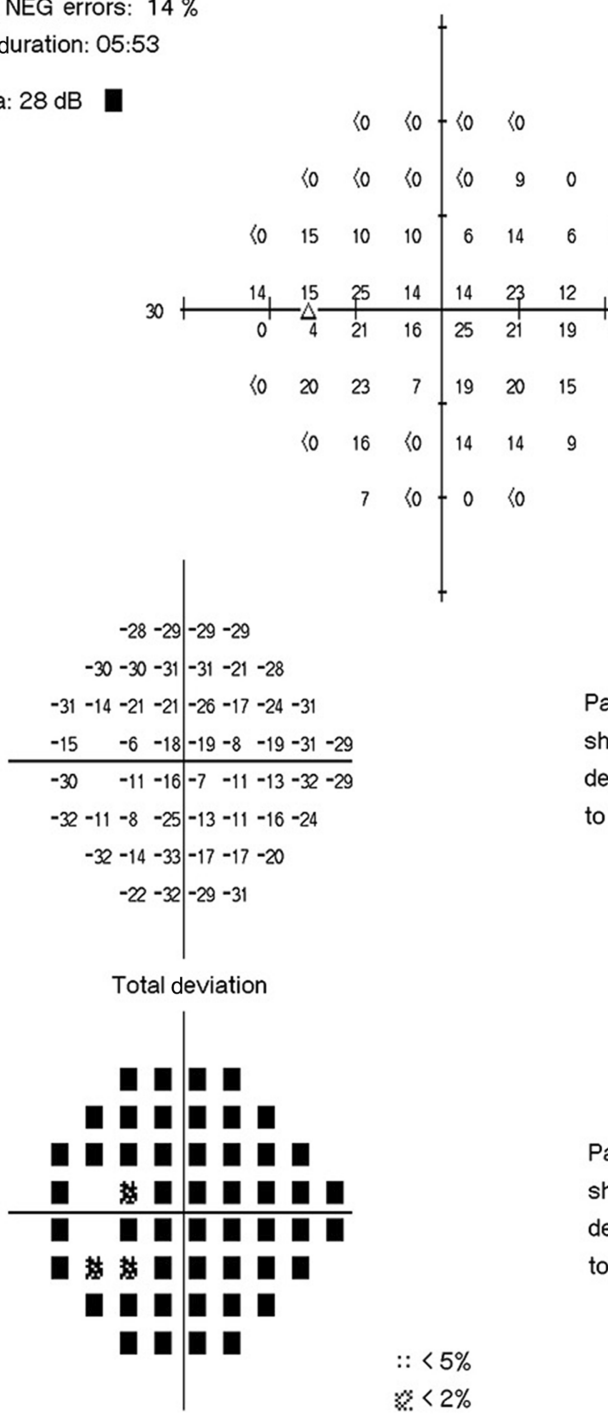

\begin{tabular}{ll|lllll}
25 & 14 & 14 & 23 & 12 & $<0$ & $<0$ \\
21 & 16 & 25 & 21 & 19 & $<0$ & $<0$
\end{tabular}

\begin{tabular}{ll|llll}
23 & 7 & 19 & 20 & 15 & 5
\end{tabular}

\begin{tabular}{lll|lll}
0 & 16 & $<0$ & 14 & 14 & 9
\end{tabular}

$<0-0<0$

$+$

更

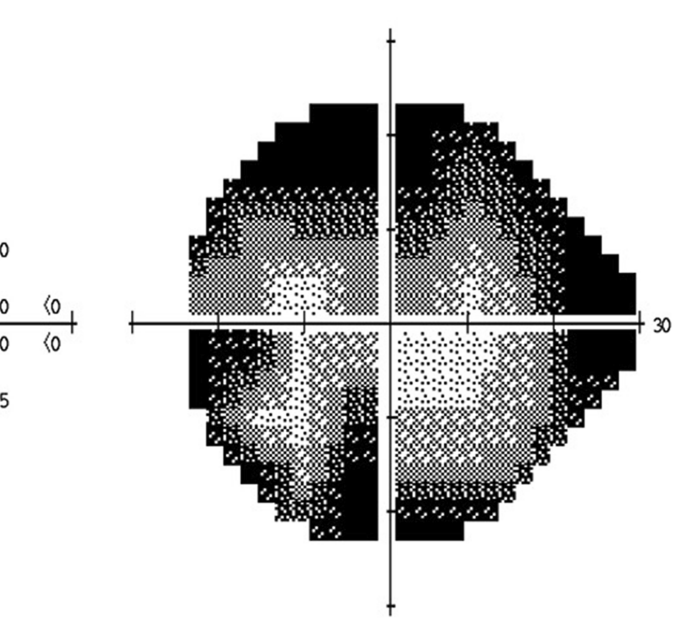

Pattern deviation not shown for severely depressed fields. refer

to total deviation.

Outside normal limits

$$
\begin{array}{ll}
\text { VFI } & 36 \% \\
\text { MD } & -21.09 \mathrm{~dB} P<0.5 \% \\
\text { PSD } & 8.54 \mathrm{~dB} P<0.5 \%
\end{array}
$$

Pattern deviation

Pattern deviation not shown for severely depressed fields. refer to total deviation.

$$
\begin{aligned}
& ::<5 \% \\
& z<2 \% \\
& z<1 \% \\
& \mathbf{0}<0.5 \%
\end{aligned}
$$

(1) 2007 Carl zeiss meditec

HFA II 750-10734-4.2.2/4.2.2

Figure 4 Continued. 
B

Single field analysis

Central 24-2 threshold test

Fixation monitor: blind spot

Fixation target: central

Fixation losses:0/13

False POS errors: $0 \%$

False NEGerrors: $8 \%$

Test duration:05:09

Fovea: $28 \mathrm{~dB}$

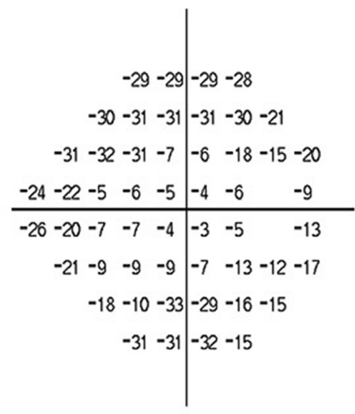

Total deviation

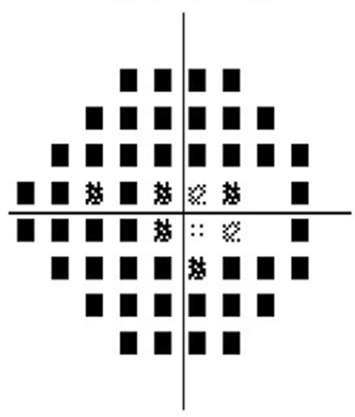

Stimulus: III, white Background: 31.5 ASB

Strategy: SITA- fast
Eye: right

$\begin{array}{ll}\text { Pupil diameter: } & \text { Date: 06-19-2017 } \\ \text { visual acuity: } & \text { Time: 1:00 PM }\end{array}$

RX: +6.25 DS -1.25 DC X 80 Age: 63 


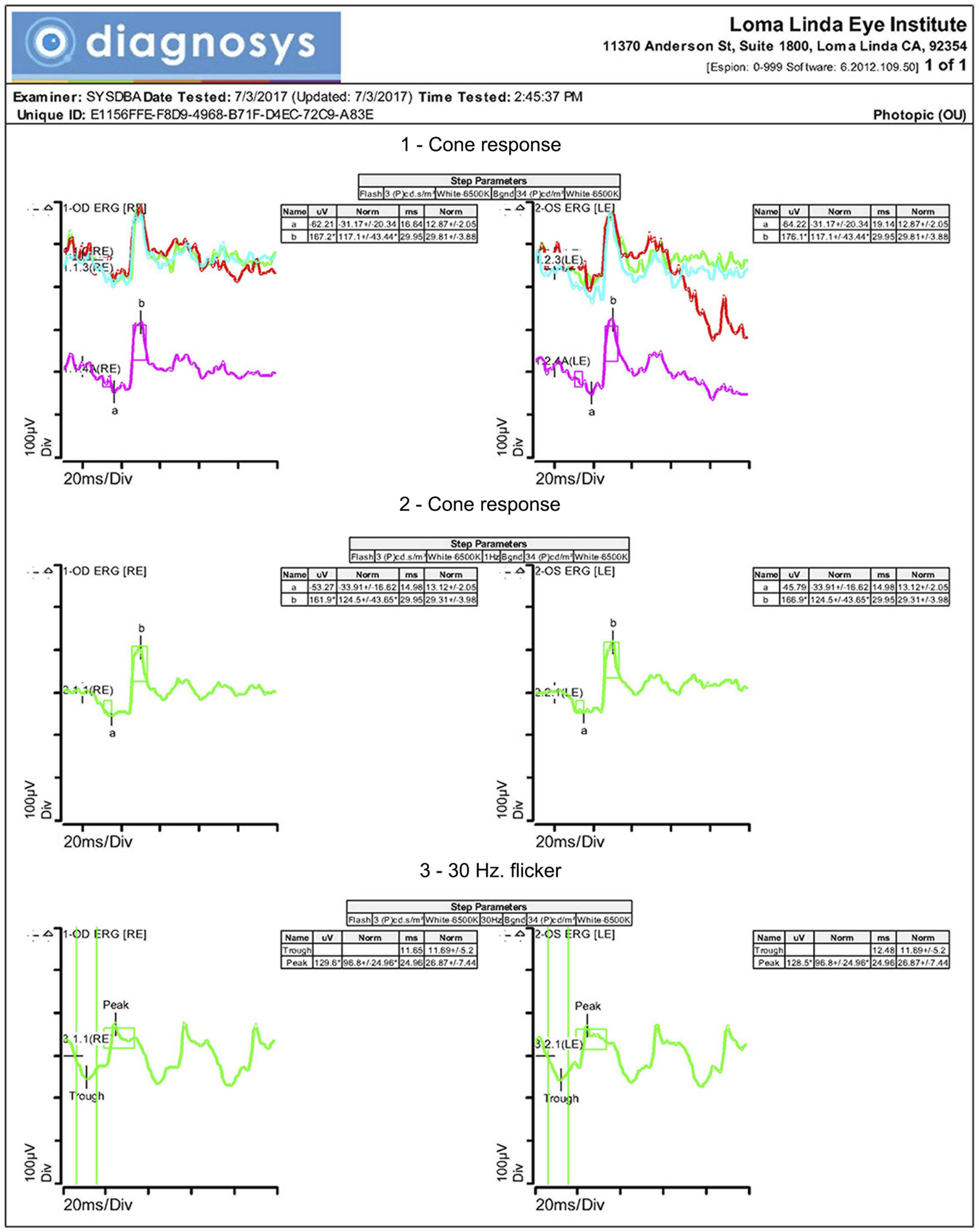

Figure 5 Continued. 


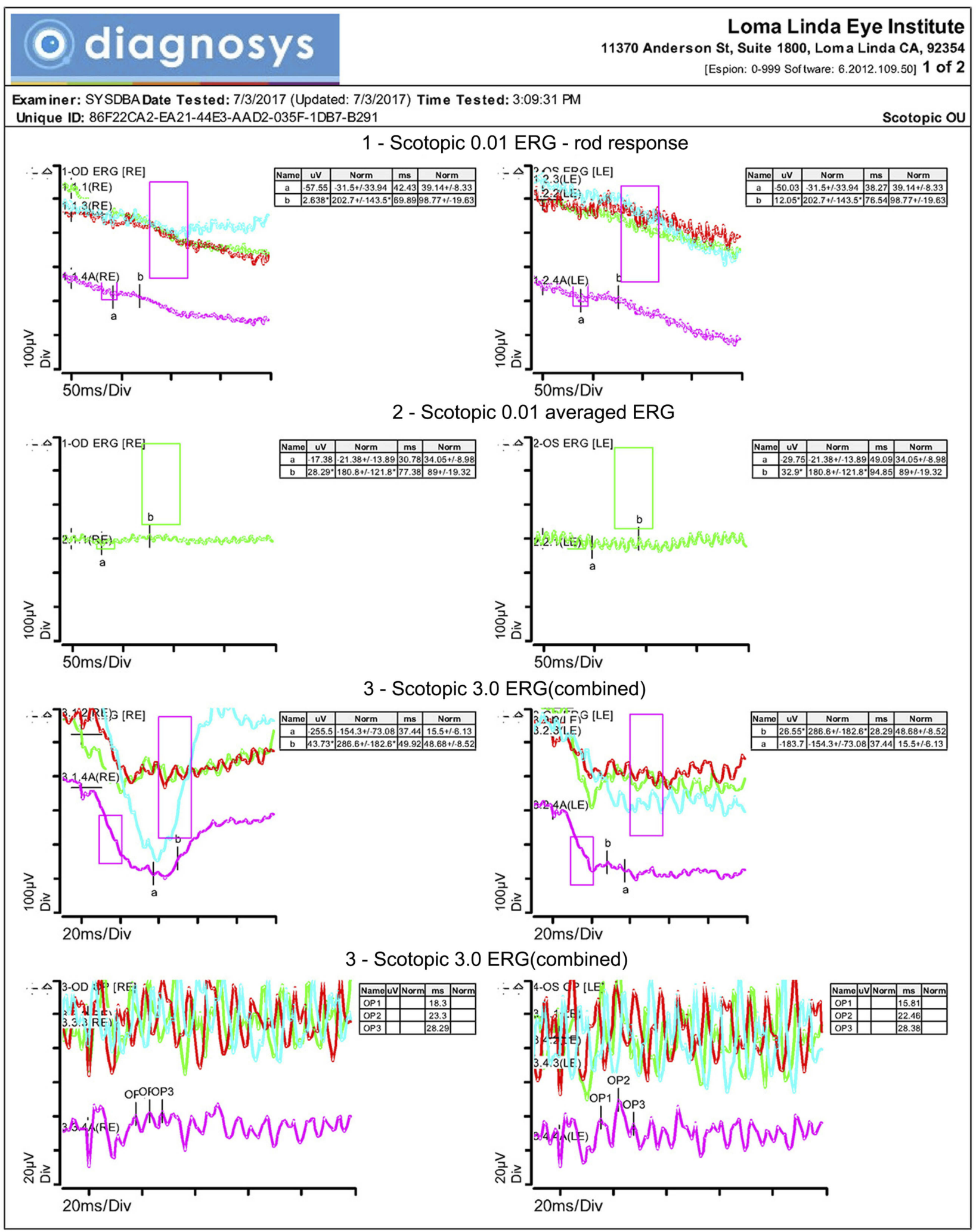

Figure 5 Continued. 


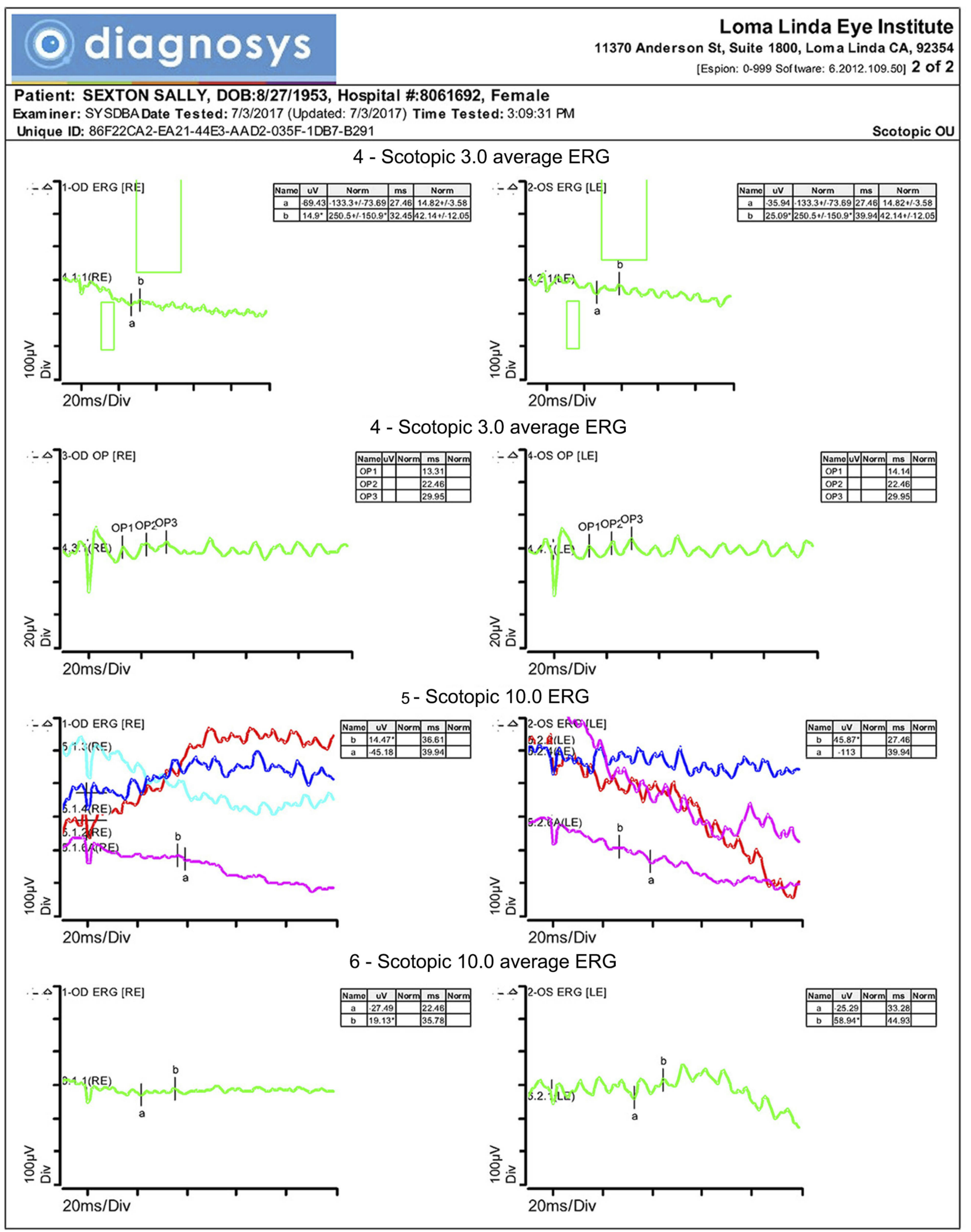

Figure 5 Full-field electroretinography: scotopic 0.01 , scotopic 3.0, oscillatory potential (OP), cone response, $30 \mathrm{~Hz}$ flicker.

Notes: Electronegative rod-system response with loss of b-wave ( 0.01 and 3$)$ and increased a-wave latency (3). Oscillatory potentials WNL. Preserved cone-system response with normal cone response, $30 \mathrm{~Hz}$ flicker. 


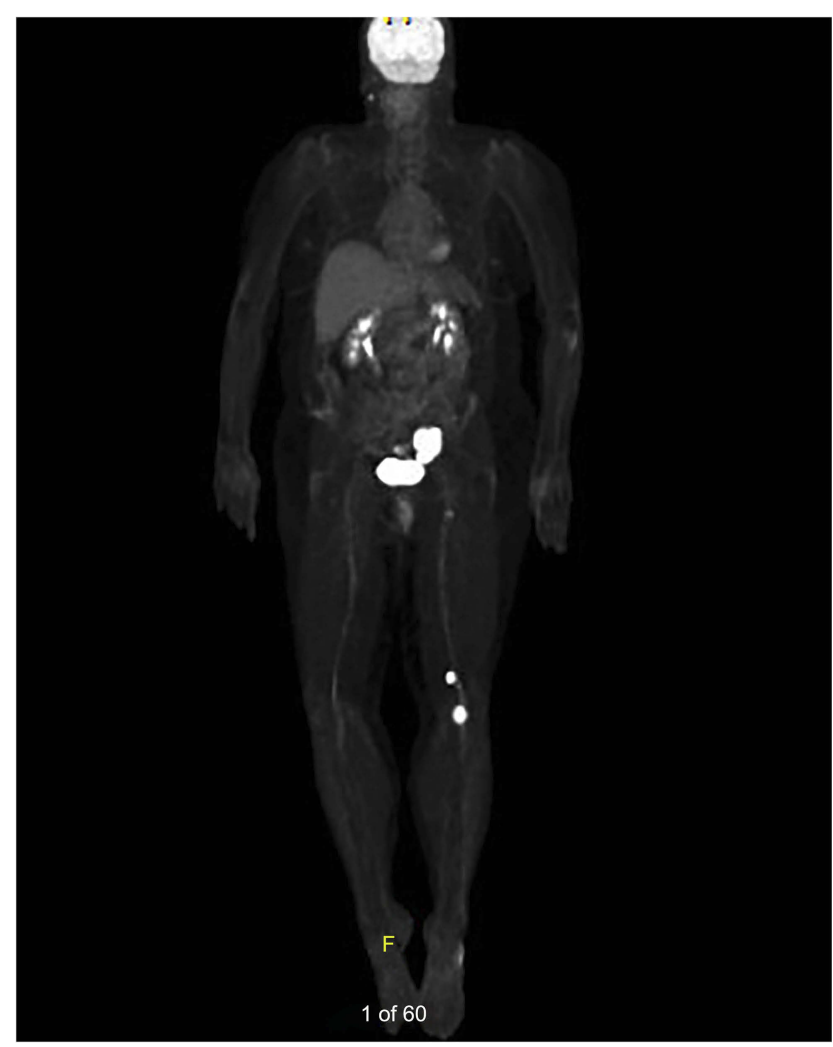

Figure 6 Whole-body nuclear medicine positron-emission tomography highlighting abnormally increased metabolic activity along the left pelvic sidewall with lesions at the left distal femur, left gastrocnemius, and left iliacus muscle.

photopsias, and loss of peripheral vision. ${ }^{1,2,4}$ As the rod system is predominantly affected and the cone system spared, both color vision and visual acuity are relatively preserved $(82 \%$ of cases with best-corrected visual acuity

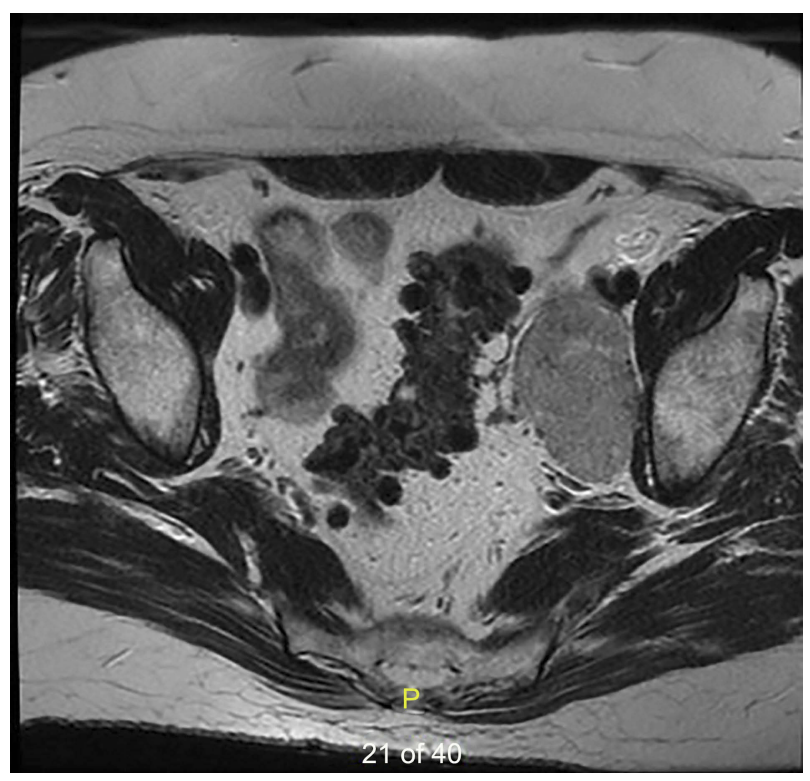

Figure 7 Magnetic resonance imaging of abdomen/pelvis without contrast, revealing large conglomerate mass along left pelvic sidewall.
$20 / 60$ or better). ${ }^{1,3}$ Visual fields show patterns of generalized constriction, paracentral scotomas, or acuate defects. ${ }^{1,4}$

ERG demonstrates a markedly reduced b-wave in the presence of a normal dark-adapted a-wave, yielding an electronegative pattern consistent with bipolar and Müller cell dysfunction. ${ }^{2,3}$ This pattern may be seen in other conditions, including congenital stationary night blindness, X-linked retinoschisis, and enhanced S-cone syndrome. Examination of serum may reveal increased aspecific antiretinal antibodies by Western blot. Postmortem staining of retinae demonstrates antibodies, particularly to bipolar cells, by indirect immunohistological studies. ${ }^{3,5}$

Management of MAR is difficult and therapeutic response variable. Options include chemotherapy with surgical cytoreduction, radiation, intravenous (IV) steroids, IVIg, and plasma exchange, ${ }^{3}$ with IVIg proving the most effective thus far, having some improved visual effect in two-thirds of patients. ${ }^{3}$ Concern remains, however, that despite its immunomodulatory effect, IVIg may increase cancer mortality, given that it decreases titers of circulating antibodies, some of which are protective against tumor spread. Current best practice includes combined cytoreductive therapy and immunomodulation. ${ }^{3}$ Overall visual prognosis is guarded, as MAR is traditionally believed to remain stable and non progressive. A minority of cases do lose central vision. ${ }^{3}$

Our case demonstrates a constellation of clinical findings that required further objective testing and critical evaluation to diagnose an ocular manifestation of a life-threatening disease. Although MAR has been described previously, this case demonstrates unique diagnostic features of MAR, including short duration of symptoms prior to diagnosis, an occult melanoma of unknown primary site, a rare metastatic location to the pelvic wall, and the presenting fundus finding of unilateral optic disk edema. This unique case of MAR demonstrates the protective role of the ophthalmologist and highlights the variable clinical presentation, given the poor prognosis and elusive nature of diagnosing melanoma.

In summary, we report a case of MAR in a female with occult melanoma. Classic ERG findings alerted prompt referral, and systemic investigation confirmed the presence of melanoma with metastasis. Written informed consent was obtained from the patient, who 
A

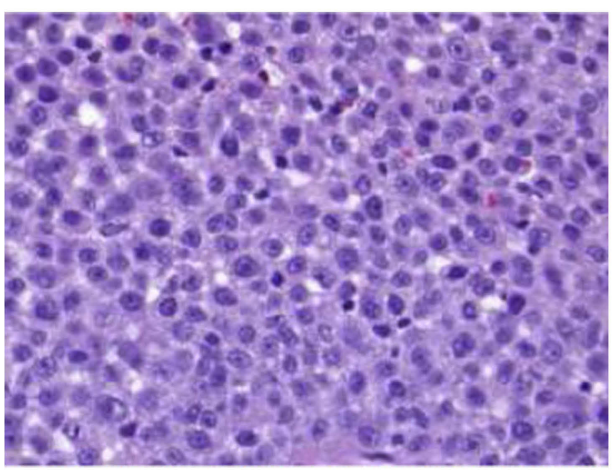

C

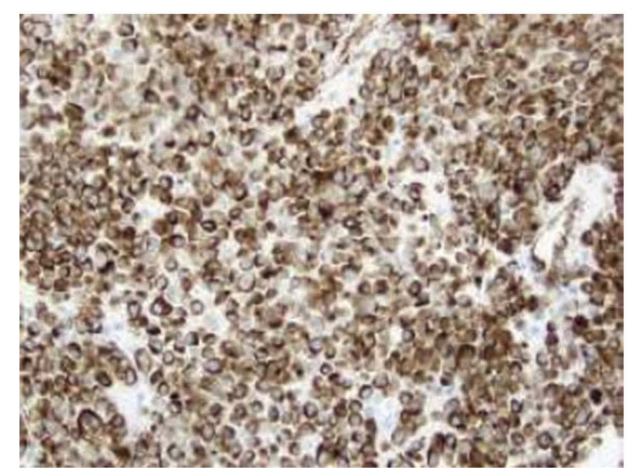

B

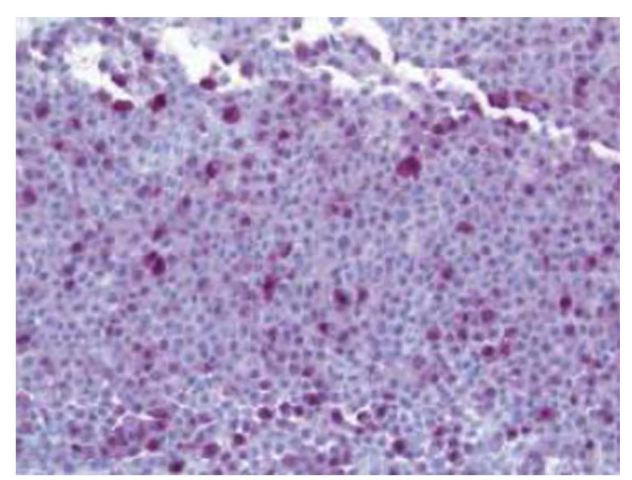

D

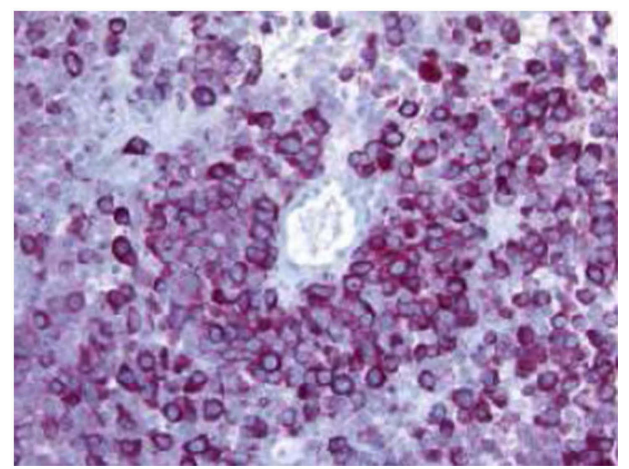

Figure 8 Histopathology of left pelvic wall mass incisional biopsy, displaying (A) sheets of large, round cells with prominent nucleoli, (B) melanoma-positive cocktail, (C) $\mathrm{SIOO}^{+}$, indicating neuroectodermal origins, and (D) $\mathrm{WTI}^{+}$cytoplasmic/golgi, consistent with a poorly differentiated melanoma.

gave permission to publish the report and accompanying figures. Institutional review board was not necessary to report the case.

\section{Disclosure}

The authors report no conflicts of interest in this work.

\section{References}

1. Boeck K, Hofmann S, Klopfer M, et al. Melanoma-associated paraneoplastic retinopathy: case report and review of the literature. $\mathrm{Br} J$ Dermatol. 1997;137(3):457-460.
2. Borkowski LM, Grover S, Fishman GA, Jampol LM. Retinal findings in melanoma-associated retinopathy. Am J Ophthalmol. 2001;132 (2):273-275. doi:10.1016/s0002-9394(01)00915-1

3. Keltner JL, Thirkill CE, Yip PT. Clinical and immunologic characteristics of melanoma-associated retinopathy syndrome: eleven new cases and a review of 51 previously published cases. $J$ Neuroophthalmol. 2001;21(3):173-187.

4. Singh AD, Milam AH, Shields CL, De Potter P, Shields JA. Melanoma-associated retinopathy. Am J Ophthalmol. 1995;119 (3):369-370. doi:10.1016/s0002-9394(14)71185-7

5. Lu Y, Jia L, He S, et al. Melanoma-associated retinopathy: a paraneoplastic autoimmune complication. Arch Ophthalmol. 2009;127 (12):1572-1580. doi:10.1001/archophthalmol.2009.311

\section{Publish your work in this journal}

The International Medical Case Reports Journal is an international, peer-reviewed open-access journal publishing original case reports from all medical specialties. Previously unpublished medical posters are also accepted relating to any area of clinical or preclinical science. Submissions should not normally exceed 2,000 words or 4 published pages including figures, diagrams and references. The manuscript management system is completely online and includes a very quick and fair peer-review system, which is all easy to use. Visit http://www.dovepress.com/testimonials.php to read real quotes from published authors. 